\title{
Solar Maximum Power Point Tracking and Its Application to Green House
}

\author{
Zakira Begum ${ }^{1}$, Mubeena Begum ${ }^{2}$, K Shiva Kumar ${ }^{3}$ \\ ${ }^{I}$ (Pg Student, DeptOfEce, Bitm College, Bellary, (Vtu Belgaum), Karnataka, India) \\ ${ }_{2}^{2}$ (Student, DeptOf It, Visvesvaraya Technological University, Belgaum, Karnataka, India) \\ ${ }_{3}^{3}$ (Assistant Professor, DeptOfEce, Bitm College, Bellary, (Vtu Belgaum), Karnataka, India)
}

\begin{abstract}
Inthisprojectalowvoltage, lowcost \&highefficiencybasedsolarmaximumpowerpointtrackingsystem forgreenhouseapplicationsis presented.Themaincontrollingelementisthemicrocontrollerprogrammeinclanguageinordertoextractmaximumsol arpowerpoint.InadditionweusebuckconverterforMPPT.Furtherthismaximumpowerisutilizedtodrivepumpsandfan sofgreenhouse.
\end{abstract}

Solar energy systems have emerged as a viable source of renewable energy over the past two or three decades, and are now widely used for a variety of industrial and domestic applications. Such systems are based on a solar collector, designed to collect the 4sun's energy and to convert it into either electrical power or thermal energy. In general, the power developed in such applications depends fundamentally upon the amount of solar energy captured by the collector, and thus the problem of developing tracking schemes capable of following the trajectory of the sun throughout the course of the day on a year-round basis has received significant coverage in this project.

This project is designed with ARM7TDMI processor. Depending upon the mode selection the data will be read by the LPC 2148 controller and the direction of the motor will be changed. With this direction the solar plates which are fixed to the stand will also rotates to gain the maximum sun rays.

\section{INTRODUCTION}

The worldconstraintsoffossil

fuelsreservesandtheeverrisingenvironmentalpollutionhaveimpelledstronglyduringlastdecadesthedevelopmentofrenewableenergysources( RES).Theneedofhavingavailablesustainableenergysystemsforreplacinggraduallyconventionalonesdemandstheim provementofstructuresofenergysupplybasedmostlyoncleanandrenewableresources. Atpresent, photovoltaic(PV)ge nerationisassumingincreasedimportanceasaRESapplicationbecauseof

distinctiveadvantagessuchassimplicityofallocation,highdependability, absenceoffuelcost,lowmaintenanceandlack ofnoiseandwearduetotheabsenceofmovingparts.Furthermore,thesolarenergycharacterizesaclean,pollutionfreeandi nexhaustibleenergysource.Inadditiontothesefactorsarethedecliningcostandpricesofsolarmodules, anincreasingeffic iencyofsolarcells,manufacturing-technologyimprovementsandeconomies of scale. Photovoltaicmodulesare interconnected assembliesof photovoltaiccells(solarcells)packagedinaweathertight housing.Modulesareratedandcalledoutbytheir wattageasmeasuredunderfactorycontrolled StandardTest Conditions (STC is equalto $1000 \mathrm{~W} / \mathrm{m} 2$ at $25^{\circ} \mathrm{C}$ and1.5atm).Inaddition,every modulehas amaximumvoltage $\left(\mathrm{V}_{\mathrm{Oc}}\right)$ andcurrent $\left(\mathrm{I}_{\mathrm{Sc}}\right)$.ThesecharacteristicsareusedbysolardesignerstocreateanefficientPV syste m.Commerciallyavailablephotovoltaicmodulescomeinthreeprimarytypes:Polycrystalline(1315\%efficiency)Amorphous(thinfilm)(5-7\%efficiency)Monocrystalline(14-17\% efficiency)

RENEWABLEENERGYIndiaisdeterminedtobecomeoneoftheworld'sleadingcleanenergyproducers. The GovernmentofIndiahasalreadymadeseveralprovisions,andestablishedmanyagenciesthatwillhelpitachieveitsgoal.R enewableEnergy,excludinglargehydroprojectsalreadyaccountsfor $\%$ ofthetotalinstalledenergycapacity,equivalent to $12,610 \mathrm{MW}$.Incombinationwithlargehydro,thecapacityismorethan $34 \%$,i.e. $48,643 \mathrm{MW}$,inatotalinstalledcapacity of 1,44,980MW.ThefollowingFig.1 showsthepercentageofrenewableenergygenerationinIndia.

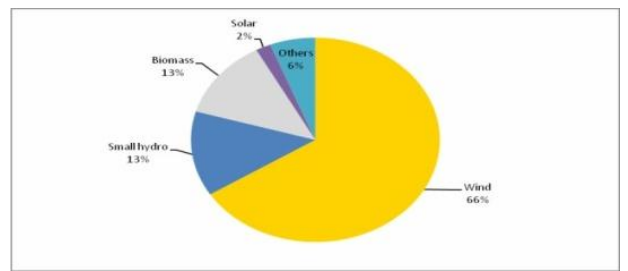

Fig.1PercentageofrenewableenergygenerationinIndia 


\section{GREENHOUSE}

Agreenhouse(alsocalleda glasshouse)isabuildinginwhichplantsare grown.Thesestructuresrangeinsize fromsmallshedstoindustrial-

sizedbuildings.Agreenhouseisastructuralbuildingwithdifferenttypesofcoveringmaterials, suchasaglass

orplasticroofand frequently glass orplasticwalls; it heatsup becauseincoming visiblesolarradiation(forwhichtheglassistransparent)fromthesunisabsorbedbyplants,soil,andotherthingsinsidetheb uilding. Airwarmedbytheheatfrom hotinterior surfacesisretained inthe buildingbytheroofandwall.In addition,thewarmedstructuresandplantsinsidethegreenhousere-

radiatesomeoftheirthermalenergyintheinfraredspectrum,to

whichglassispartlyopaque,sosomeofthisenergyisalsotrappedinsidetheglasshouse.

\section{PHOTOVOLTAICEFFECT}

Whenirradiancehitsthesurfaceofsolar

cell,anelectricalfieldisgeneratedinsidethecell.AsseeninFig.2,thisprocessseparatespositiveandnegativechargecarrie rsinanabsorbingmaterial(joiningp-typeandn-

type).Inthepresenceofanelectricfield, thesechargescanproduceacurrentthatcanbeusedinanexternalcircuit. Thisgener atedcurrent depends ontheintensityoftheincident radiation.Thehigherthelevel oflight intensity,themoreelectrons canbeunleashedfromthesurface, themorecurrentisgenerated.Thedesignofeffectiveandprofitablefacilitiesonthebasis ofsolarmodules isespeciallyimportant. Software dedicated to thesimulationofphotovoltaic systems canrealizeextensiveandpreciseanalyzes, buttheygenerallydonotallowtheusertomodifythealgorithms.

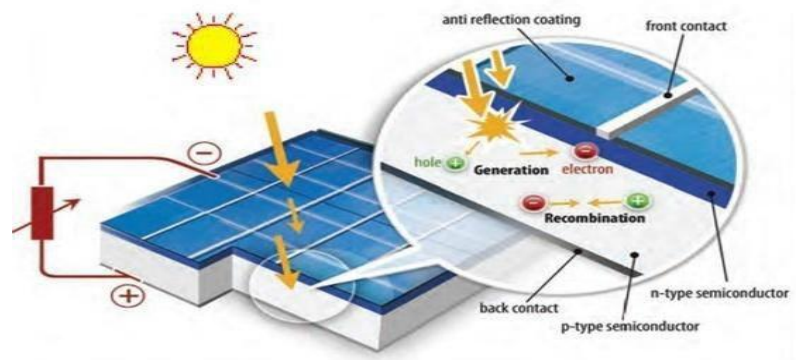

Fig.2SchematicCross-SectionofaTypicalSolarCell

\section{MAXIMUMPOWERPOINTTRACKING}

Thepeakpowerisreachedwiththehelpofmanualtrackingorautomatictracking.Manualtrackingisnotpossibles oautomatictrackingispreferredtomanualtracking.Anautomatictrackingcanbeperformedbyutilizingvariousa lgorithms.

(i) PerturbandObserve

(ii) IncrementalConductance

(iii) Constant Voltage Method

Thealgorithmsareimplementedinamicrocontrollerorapersonalcomputertoimplementmaximumpowertrack ing[4].Thealgorithmmaychangethedutycycleofthedc/dcconvertertomaximizethepoweroutputofthemodule andmake it operateatthepeakpower point of themodule.Similarlyinvertermodulationcanbe varied andthemaximumpowerpointcanbeobtained.

\section{METHODOLOGY}

Theprototype modelofasolarmicrocontrollerbasedgreenhouse workingonbasisofmaximumpowertrackingwillbemadeinthefollowingsteps:

- Completelayoutofthe wholesetup willbe drawninformofablockdiagram.

- Dayandnightsensor willfirstsense theconditionandgiveitsoutputtothemicrocontroller.

- The photovoltaic panelwill

bemounted atanoptimumanglefromtheverticalasonad.c.motordrivenbyadriverI.C.suchthatthepanelmovesandthecontrolle rcheckstheoutputvoltageatvariouspoints.

- Identificationofpointswheremaximumvoltageandhencemaximumpowerreceivedbythesolarpanel.

- Maximumpower willbedetectedbyMPPTAlgorithmdeveloped,then fed toAnalogto Digital converterandstoredinmicrocontroller.

- Themotorandhencethepanelwill bestoppedwhenmaximum powerwill bereceivedby thesolar panelandhencewillstartchargingthebattery.

- A checkonsoil,humidityandtemperaturecanalsobekeptusingsensors andsolarenergystoredcanbeusedtoruna waterpumpand water airpumpsoasto maintaindifferentparametersatanoptimumlevel[5]. 


\section{LITERATURE SURVEY:}

The research work is going on for tracking maximum solar power. In this project MPPT algorithm is used for tracking maximum power and ARM controller is used for regular monitoring of the environmental conditions of green house and also provides the necessary precautions to be taken for yield to increase. As irrigation becomes the major part to have growth at economic status of our country, this became quite interesting concept which is going to be major tool to reduce the power cost required for automization of agriculture.

Hence with this project implementation we can generate the power \& the generated power is used by ARM controller for controlling the environmental conditions of green house. Further the generated power can be used for the other real time applications.

\section{FLOW CHART}

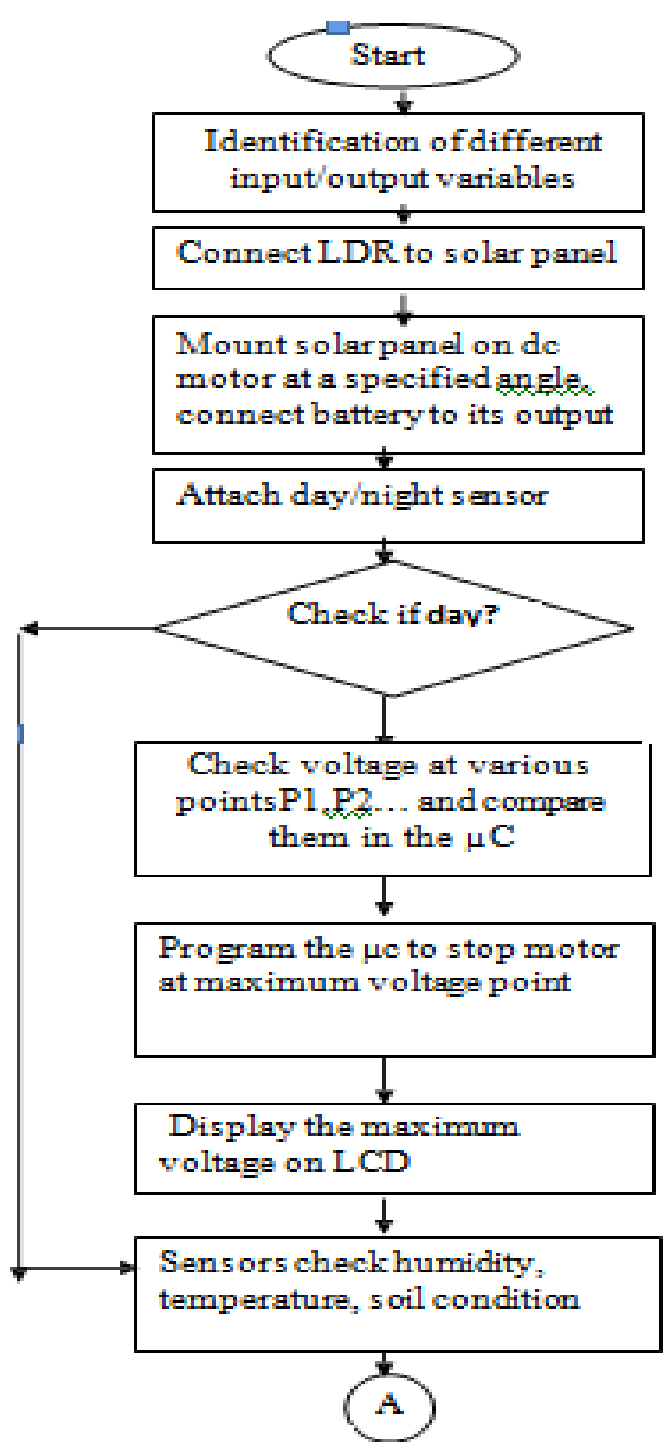




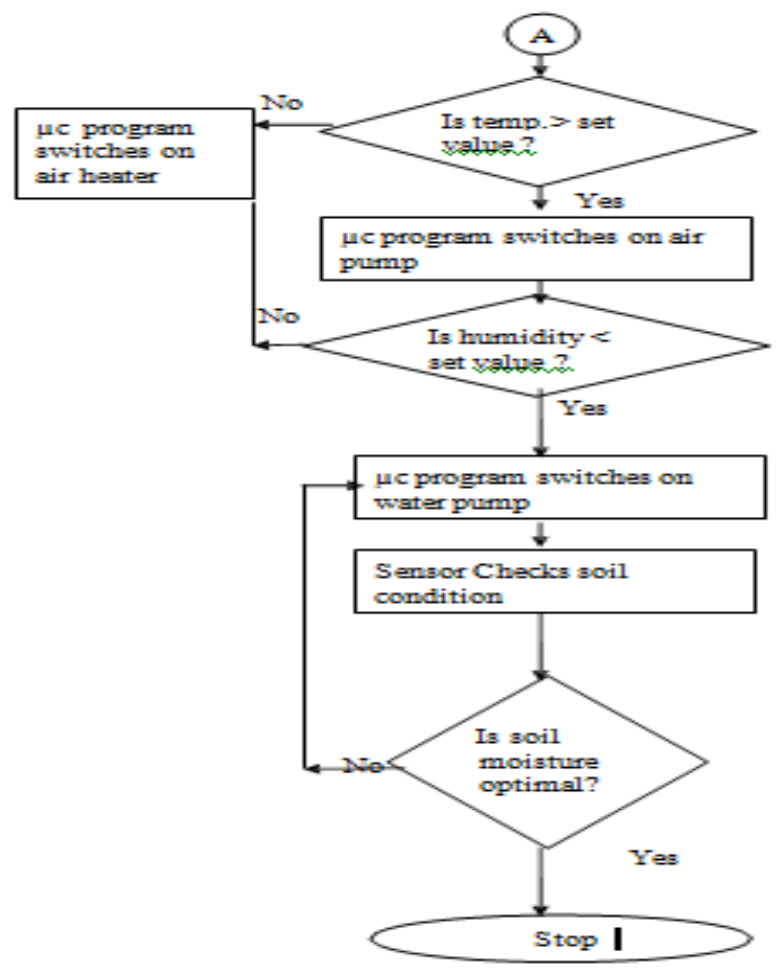

\section{BLOCK DIAGRAM}

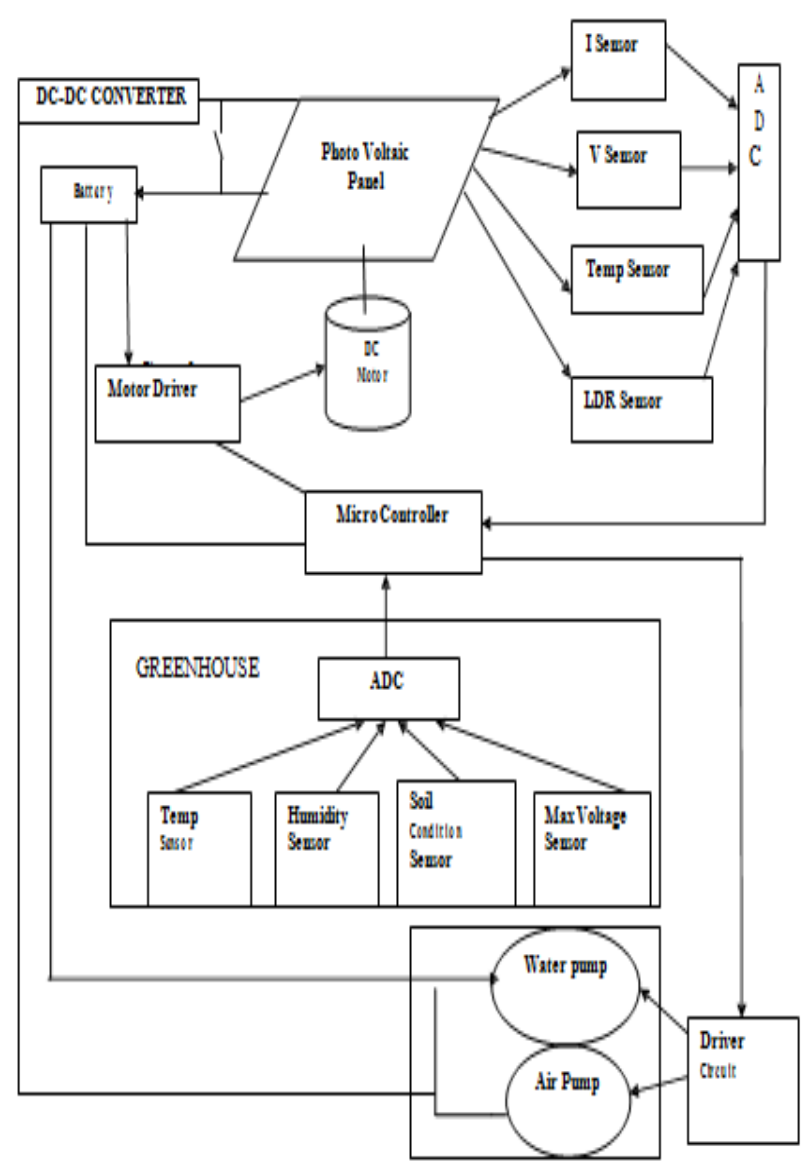

$\cos 0$ 


\section{HARDWARE DESCRIPTION}

[1]. SolarPanel:Itisapackaged,connectedassemblyofphotovoltaiccells.Herea17V10Wsolarpanelwillbeused.
[2]. DeMotor(20rpm):Adc motoris amechanicallycommutated electricmotorpoweredfromdirectcurrent $(\mathrm{dc})$.ItisusedforrotatingtheSolarPanelaccordingtosunlight.

[3]. Relay(6Vdc):Arelayisanelectricallyoperatedswitch.Manyrelaysusean magnettooperateaswitchingmechanismmechanically.Relaysareused whereitisnecessarytocontrolacircuitbyalo w-

powersignal(withcompleteelectricalisolationbetweencontrolandcontrolledcircuits),orwhereseveralcircuitsmu stbecontrolledbyonesignal.Hereitisused forcontrollingwaterpumpand waterairpump.

[4]. Sensors:Temperaturesensorsaredevicesusedtomeasurethetemperatureofamedium. Therearetwokindsoftempe raturesensorscontactsensorsandnon-contactsensors.

However,thethreemaintypesarethermometers,resistancetemperaturedetectorsandthermocouples.Allthreeofth esesensors

measureaphysicalproperty(i.e.volumeofaliquid,currentthroughawire), whichchangesasafunctionoftemperatur e.

[5]. HumiditySensor:isthepresenceofwaterinair.Theamountofwatervaporinaircanaffecttheplantsgrowth.Thepres enceofwatervaporalsoinfluencesvariousphysical,chemical,andbiologicalprocesses.

[6]. DayandNightSensor:Aphotoresistororlightdependentresistor(LDR) resistancedecreaseswith increasingincident exhibitsphotoconductivity.Aphotoresistor ismade isaresistorwhose ofahighresistancesemiconductor.high frequency,photonsabsorbedbythesemi-conductorgiveboundelectronsenoughenergytojumpintothe conductionband.Theresultingfreeelectron(anditsholepartner)conductelectricity,therebyloweringresistance.

[7]. ADC0808/ADC0809:It'sa8-Bit $\mu$ PcompatibleA/Dconverterswith8channelmultiplexer.TheADC0808,ADC0809dataacquisitioncomponentisamonolithicCMOSdevicewithan8bitanalog-to-digitalconverter,8-channelmultiplexerandmicroprocessorcompatiblecontrollogic.The8bitA/Dconverterusessuccessiveapproximationastheconversiontechnique.Theconverterfeaturesahighimpedan cechopperstabilizedcomparator,a256Rvoltagedividerwith analogswitchtreeandasuccessiveapproximationregister.The8-channelmultiplexercandirectlyaccessanyof8single-endedanalogsignals. Thedeviceeliminates theneedforexternalzeroandfullscaleadjustments.Easyinterfacingtomicroprocessorsisprovidedbythelatchedanddecodedmultiplexeraddressin putsandlatchedTTLTRI-STATE®outputs.

[8]. 8. KIA7805AP:ItisathreeterminalpositivevoltageregulatorsuitableforCMOS,TTLandotherdigitalIC'spower supply. It has internal thermal overload protection and internalshortcircuitcurrentlimitingfeatures.

[9]. 9.

Arm

controller:Arm controller(LPC2148)isusedtocontrolandinterfacetheRelays,SensorsandMotor.Theitisalow-power,highperformance controller.

- Highly sensitive

\section{ADVANTAGES}

- Works according to the sun direction

- Fit and Forget system

- $\quad$ Night - Day mode sensing

- Low cost and reliable circuit

- Complete elimination of manpower

- Street lights

VIII. APPLICATIONS

- Garden Lights

- Solar water heater

- Hotels, hostels and house hold applications

- Offices

- Industries 


\section{IX.CONCULSION}

- Aftercarryingouttheproposedwork,theinvestigatorexpectsthefollowingoutcomes:

- Developmentofasolargreenhousebasedonmaximumsolarpowertrackingwithvariousparametersbeingcontrolle dbyamicrocontrollerandmaintainedfurthertoanoptimumvaluerequiredforadequategrowthofplant;using thestoredsolarenergyonly .Storageofsolarenergy in abatteryandthen its re-utilizationfordriving awaterpumpandawaterairpumpto maintainoptimummoistureandtemperaturerequiredforadequategrowthofplant.

- Anincreaseinoutputofsolarpanelduetotheimplementationofmaximumsolarpowertracking.Theproposedgreenh ouseisveryusefulfor hillyareas wherethere isscarcesunlightin winters.Theproposedgreenhousewillconsistofminimumhardware.Andwillhaveveryfastresponseandwilloccup yminimumspaceItcanbebuiltevenontherooftopofhouses, inhighlypopulatedhillyareas.Energycanalsobestored atalargescaleandmaybeutilizedforheatingthehousewaterinwintersavailableintanksofhouse.

\section{REFERENCES}

[1]. I.H.Altas,A.M.Sharaf,"APhotovoltaicArraySimulation Model for MATLAB-SIMULINK GUIEnvironment," inProceedingsof theInternational ConferenceonClean Electrical Power,pp.341-345, 2007.

[2]. E.Koutroulis,K.Kalaitzakis,N.C.Voulgaris, “DevelopmentofaMicrocontroller-

BasedPhotovoltaicMaximumPowerPointTrackingControl System”,IEEETransactionsonPower Electronics,Vol. 16,No1,pp.4654,January2001.

[3]. G.Petrone,G.Spagnuolo,R. Teodorescu,M. Veerachary,M. Vitelli,"ReliabilityIssuesin PhotovoltaicPowerProcessingSystems, "IEEETransaction.on Industrial Electronics, Vol.55,no.7,pp.2569-2580,July2008

[4]. JaenC,MoyanoC,SantacruzX,PouJ,AriasA,"OverviewofMaximumPowerPointTrackingControltechniquesusedinPhotovoltaicSystems" ,15thIEEEInternational ConferenceonElectronics,,CircuitsandSystems,ICECSpp 1099 - 11025,2008.”.

[5]. VeeracharyM,SenjyuT,UezatoK,"Voltage-BasedMaximumPowerPointTrackingControlofPVSystem",IEEETransactionsonAerospace and ElectronicSystems", $\quad$ Vol.38,No.1,pp.262-270,6January2002.

[6]. DavidSanzMorales,"MaximumPowerPointTrackingAlgorithmsforPhotovoltaicApplications",onlinejournalofwww.ijera.com,2010.

[7]. AsmarashidPonniran,AmmarHashim,AriffuddinJoret,“ADesignof LowPower SingleAxisSolar TrackingSystem Regardless ofMotor Speed”,International Journal ofIntegratedEngineering,Vol.3No.3 online24December,2011.

[8]. BasimAlsayid,"Modeling andSimulation of Photovoltaic Cell/Module/Array withTwo-Diode Model",International Journal ofComputerTechnologyand ElectronicsEngineering(IJCTEE)Volume1,Issue 3,June,2012.

[9]. Joe-Air Jiang,Tsong-LiangHuang,Ying-Tung Hsiao and Chia-Hong Chen,"MaximumPowerTracking forPhotovoltaicPowerSystems",Tamkang Journal of Science andEngineering,Vol. 8,No 2,pp.147-153(2005)

[10]. Kassem,A,Hamad,M.,Amicrocontroller-basedmulti-function solar tracking system,IEEEInternationalSystemsConference(SysCon),47 April 2011

\section{BIBLOGRAPHY}

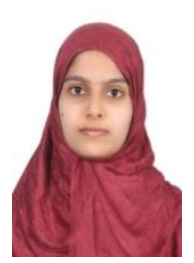

\section{AUTHOR 1: ZAKIRABEGUM}

Pursuing M.Tech (VLSI) student of BITM College,BELLARY,Karnataka and completed $\mathrm{BE}(\mathrm{IT})$ in PDIT College HOSPET, Karnataka of VisvesvarayaTechnologicalUniversity,Belgaum,Karnataka, INDIA.area of interest in embedded systems, vlsi design, control system,Biomedicals.

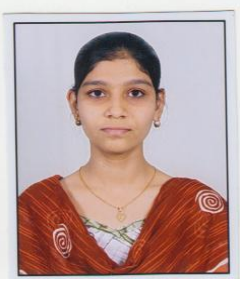

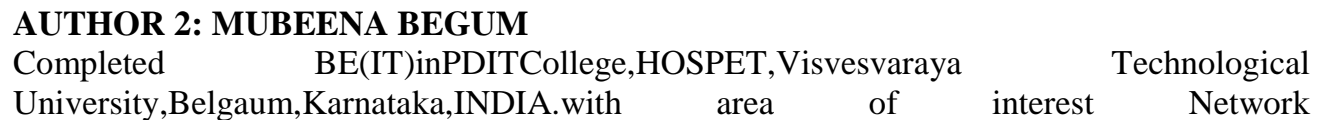
analysis,controlsystem,Biomedicals, VHDL.

\section{AUTHOR3: K SHIVA KUMAR}

Obtained MTECH(CNE) in BITM COLEGE,BELLARY,Karnataka,INDIA and obtained BE(ECE) in RYMEC, BELLARY, Karnataka of Visvesvaraya Technological University, Belgaum, Karnataka, INDIA working as Assistant Professor in BITM COLLEGE, BELLARY. With working experience of 14 years in teaching field with area of interest in embedded systems, operating systems, Computer Networks, Analog\& digital communication. 